\title{
The Positive Predictive Value of a Hyperkalemia Diagnosis in Automated Health Care Data
}

\author{
Marsha A. Raebel, PharmD ${ }^{1,2,3}$, Michael L. Smith, JD, PharmD ${ }^{2,4}$, Gwyn Saylor, BA ${ }^{1}$, Leslie \\ A. Wright, MA ${ }^{1}$, Craig Cheetham, PharmD, $\mathbf{M S}^{3,5}$, Christopher M. Blanchette, PhD $^{6,7,8}$, and \\ Stanley $\mathbf{X u}, \mathbf{P h D}^{1}$ \\ ${ }^{1}$ Kaiser Permanente Colorado Institute for Health Research, Denver, Colorado \\ ${ }^{2}$ School of Pharmacy, University of Colorado at Denver, Denver, Colorado \\ ${ }^{3} \mathrm{HMO}$ Research Network Center for Education and Research in Therapeutics (CERTs) \\ ${ }^{4}$ Kaiser Permanente Colorado Department of Pharmacy Services, Denver, Colorado \\ ${ }^{5}$ Pharmacy Analytic Services, Kaiser Permanente Southern California, Downey, California \\ ${ }^{6}$ Lovelace Respiratory Research Institute, Kannapolis, North Carolina \\ ${ }^{7}$ School of Pharmacy, University of North Carolina at Chapel Hill, Chapel Hill, North Carolina \\ ${ }^{8}$ Salisbury VAMC, Salisbury, North Carolina
}

\begin{abstract}
Purpose-Our objectives were to determine performance of coded hyperkalemia diagnosis at identifying 1) clinically-evident hyperkalemia and 2) serum potassium $\geq 6 \mathrm{mmol} /$ liter.

Methods-This retrospective observational study included 8,722 patients with diabetes within an integrated healthcare system who newly-initiated an angiotensin converting enzyme inhibitor, angiotensin receptor blocker, or spironolactone. The primary outcome was first hyperkalemiaassociated event (hospitalization, emergency department visit or death within 24 hours of coded diagnosis and/or potassium $\geq 6 \mathrm{mmol} /$ liter) during the first year of therapy. Medical records were reviewed.
\end{abstract}

Results-Among a random sample of 99 patients not coded as having hyperkalemia, none had hyperkalemia upon record review. Among all 64 patients identified as having hyperkalemia, all had hospitalization or emergency department visit associated with coded diagnosis or elevated potassium. Of 55 with coded diagnosis, 42 (PPV 76\%) had clinically-evident hyperkalemia; 32 (PPV 58\%) had potassium $\geq 6$. Of 9 identified using only potassium $\geq 6,7$ (PPV 78\%) had clinically-evident hyperkalemia.

Conclusions-Nearly one-fourth of patients with coded diagnosis do not have clinically-evident hyperkalemia and nearly one-half do not have potassium $\geq 6$. Because both false positives and negatives occur with coded diagnoses, medical record validation of hyperkalemia-associated outcomes is necessary.

\section{Keywords}

Hyperkalemia; positive predictive value; sensitivity; specificity; ACEi; ARB

Corresponding Author/Address: Marsha A. Raebel, PharmD, Institute for Health Research, Kaiser Permanente Colorado, PO Box 378066, Denver, CO 80237-8066, Phone: (303) 614-1260, Fax: (303) 614-1265, Marsha.A.Raebel@kp.org. 


\section{Key Points}

- Automated healthcare data can be useful when identifying hyperkalemiaassociated adverse outcomes. Serum potassium laboratory test results provide incremental value in identifying such outcomes. However, no single automated data definition of hyperkalemia-associated outcome identifies all patients who experience clinically-evident hyperkalemia.

- Using coded diagnosis alone without including available serum potassium concentration information underestimates the number of patients with clinicallyevident outcomes. The combination of coded diagnosis and serum potassium concentration $\geq 6 \mathrm{mmol} /$ liter also underestimates the number of patients with clinically-evident outcomes.

- Nearly one-fourth of patients with coded hyperkalemia diagnosis do not have clinically-evident hyperkalemia and nearly one-half do not have serum potassium concentration $\geq 6 \mathrm{mmol} /$ Liter.

- Hyperkalemia was not coded as a cause of death. Death certificates were not useful in identifying patients in whom hyperkalemia may have been a contributing cause of death.

- Because both false positives and negatives occur with coded hyperkalemia diagnosis in administrative data, medical record validation of hyperkalemia outcomes is necessary.

\section{Introduction}

Hyperkalemia is an uncommon, potentially fatal consequence of therapies such as angiotensin converting enzyme inhibitors (ACEi), angiotensin receptor blockers (ARB), potassium-sparing diuretics, potassium supplements, beta-blockers, and nonsteroidal antiinflammatory agents. ${ }^{1-7}$ Serious (> $6 \mathrm{mmol} /$ liter) and severe (> $7 \mathrm{mmol} /$ liter) hyperkalemia can be life-threatening, but even severe hyperkalemia can sometimes go unrecognized with few symptoms prior to cardiac arrest. ${ }^{8,9}$

The positive predictive value of the International Classification of Diseases, Ninth Revision (ICD-9) hyperkalemia code has not been evaluated. Further, the proportion of coded diagnoses associated with elevated potassium concentrations and corresponding signs and symptoms has not been sufficiently assessed. The performance of the hyperkalemia code is important because misclassification resulting in under- or overestimation of outcomes would occur if clinically-evident hyperkalemia was present but not coded or coded but only incidental to the medical visit. The aims of this study were to determine the performance of a coded hyperkalemia diagnosis in administrative data at identifying patients with clinicallyevident hyperkalemia and serum potassium $\geq 6 \mathrm{mmol} / \mathrm{liter}$.

\section{Methods}

This study was conducted using the patient cohort from one of the three integrated healthcare delivery systems involved in a large retrospective observational study of serious hyperkalemia among ambulatory patients with diabetes newly-initiating ACEi, ARB, and or spironolactone. ${ }^{10-12}$ The KPCO Institutional Review Board approved this study and waived the informed consent requirement. 
This study cohort included patients with diabetes without end-stage renal disease (ESRD) who were Kaiser Permanente Colorado (KPCO) members with pharmacy benefits for at least 12 months between January 1, 2001 and December 31, 2006. These 8,722 individuals averaged 61.5 (SD 13.4) years of age and included 4,147 females (47.6\%). Most patients were initially dispensed an ACEi $(n=8,085 ;$ ARB $n=447$; spironolactone $n=136$; combination $n=54$ ) and remained on the initial therapy for a mean of 227 (SD 139) days. Patients were censored from the study at the last day of the dispensed days' supply, death, first hyperkalemia outcome, ESRD diagnosis, disenrollment, or end of the study.

The primary outcome was the first hyperkalemia-associated event, defined as a) potassium concentration $\geq 6 \mathrm{mmol} /$ liter, b) coded diagnosis of hyperkalemia (ICD9 276.7), or c) combination of "a" and "b" co-occurring within 24 hours of an inpatient (IP) or emergency department (ED) claim or death (ICD10 code) within 365 days after initiating therapy. We previously determined the sensitivity (SE) and specificity (SP) of KPCO administrative data to be excellent at identifying both drug dispensing and potassium monitoring. ${ }^{13}$ To ensure capture of deaths, we accessed disenrollment records, hospital discharge status, the KPCO mortality database, and death certificates.

To enable calculation of the positive predictive values (PPV) of a hyperkalemia diagnosis in automated data at identifying patients with 1) clinically-evident hyperkalemia and 2) potassium $>6$, the medical records of all patients identified as meeting the outcome definition were requested for review. The medical record was considered the gold standard. To estimate the SE and SP of automated data at identifying hyperkalemia, we assumed that reviewing records of a random sample of 100 patients identified from automated data as not having hyperkalemia represented the population. Abstractions were conducted by one clinical pharmacist (MLS). As a quality check, five percent of abstractions were reabstracted by the lead investigator (MAR).

Patients were considered to have clinically-evident hyperkalemia when any of the following were documented:

- Signs: Complete or other atrioventricular (AV) block, AV dissociation, paroxysmal ventricular tachycardia, ventricular fibrillation/flutter, cardiac arrest, bradycardia, other dysrhythmias, insertion of a temporary pacemaker.

- Treatment: Potassium binding resin, intravenous glucose plus insulin, intravenous calcium, hydration, acute hemodialysis.

- Change in clinical plan: Discontinuation or adjustment in ACEi/ARB/ spironolactone dosage/frequency, change to another ACEi/ARB/spironolactone, order for additional potassium monitoring, other (e.g., discontinuing potassium supplement).

We obtained data from pharmacy, diagnosis, laboratory, and other KPCO databases, ambulatory electronic medical records (EMR), ED or IP medical records, and death certificates. In analyzing the random sample of patients identified as not experiencing hyperkalemia, we encountered zero counts. Because zero counts do not necessarily imply no risk, ${ }^{14}$ to quantify uncertainty, exact binomial confidence intervals were constructed with permutations. ${ }^{15}$ All data checks and analyses were performed with SAS version 9.1 (SAS Institute Inc., Cary, North Carolina).

\section{Results}

Among the 8,722 patients, $71(0.8 \%)$ experienced an IP or ED visit associated with potassium $\geq 6.0 \mathrm{mmol} / \mathrm{L}$ and/or a coded hyperkalemia diagnosis occurring within 24 hours 
of each other (Figure). Ambulatory EMR information was available for all 71 patients and for the 100 randomly-sampled patients. IP/ED information was not available for six (Figure), that is, we accessed comprehensive records for 96.5\% (165 of 171). During record review, two patients were identified as prevalent medication users and excluded. The final number of patients included in analysis was 64 with and 99 without outcome.

Of the 99 patients identified in administrative data as not having a hyperkalemia outcome, none had hyperkalemia upon record review (Figure, Table). Of the 64 patients identified in administrative data as having hyperkalemia (Figure), all had an IP/ED visit associated with hyperkalemia (Table). Overall, 49 of 64 (PPV 77\%) had clinical signs of, received treatment for, and/or had a change in clinical plan associated with hyperkalemia (Table), including 42 of 55 (PPV 76\%) with coded diagnosis. Thirty-two of these 55 (PPV 58\%) had potassium $\geq$ 6 documented.

When considering the highest potassium at the time the patient experienced hyperkalemia, all 9 who had an IP/ED visit plus potassium $\geq 6$ without coded diagnosis had documented potassium $\geq 6$. Twenty of 41 who had a medical visit and coded diagnosis also had potassium $\geq 6$ documented, but for others in this subgroup, the highest potassium identified was between $5.5-5.9$ (12 patients) or $\leq 5.4$ ( 9 patients). Among 14 who met outcome criteria based on medical visit, coded diagnosis and potassium $\geq 6$, when the record was reviewed, 12 had documented potassium $\geq 6$, while two had potassium between 5.5 and 5.9.

No patient had a coded diagnosis of hyperkalemia-associated death. However, five whose charts were reviewed died at the time of the hyperkalemia, as did one whose chart was unavailable. In three, the visit note stated that hyperkalemia likely contributed to death (e.g., "cardiac arrest, likely due to hyperkalemia"). Hyperkalemia was not mentioned on the death certificate of any patient.

\section{Discussion}

Our study demonstrates that no single automated data definition of hyperkalemia-associated outcome identifies all patients who experience clinically-evident hyperkalemia. Using coded diagnosis without including potassium concentration information underestimates the number of patients with clinically-evident outcomes: Seven of 49 symptomatic patients would have been misclassified if we had not incorporated potassium concentration data. Nine patients who had potassium $\geq 6$ did not have a coded diagnosis; seven of these had clinically-evident hyperkalemia. Conversely, although one might think the combination of coded diagnosis plus potassium $\geq 6$ would optimize identifying clinically-evident hyperkalemia, requiring the combination would have only identified 12 of 49 symptomatic patients.

Our results also demonstrate that the PPV of the diagnosis code is very good (PPV 77\%) at identifying clinically-evident hyperkalemia, but performs less well at identifying patients with potassium $\geq 6$ (PPV 58\%). Finally, we found that the hyperkalemia death code and death certificates were not useful in identifying patients in whom hyperkalemia may have contributed to death.

Concern about outcomes misclassification was the basis for this study. We found that 15 (of 64) patients identified with hyperkalemia from administrative data did not have clinical signs or treatment or a change in clinical plan, including 13 (of 55) with coded diagnosis. Some think that if clinical signs and treatment are lacking, a hyperkalemia diagnosis should not be coded. However, others think that when potassium is $>5.0$, hyperkalemia should be coded. If we had used the latter value, one patient would have been considered misclassified. However, we used the stringent requirement of potassium $\geq 6$ that resulted in 23 being considered misclassified. 
This work presents a picture of hyperkalemia among patients with diabetes treated with $\mathrm{ACEi}, \mathrm{ARB}$, or spironolactone in a real-world setting. To determine the performance and validity of automated data, we abstracted records of all patients identified as having a hyperkalemia-associated IP/ED visit or death. We applied consistent definitions and accessed comprehensive databases. The net result is that we present an inclusive clinical and administrative picture of patients with serious hyperkalemia outcomes.

The project was conducted at one healthcare system; the results may not be fully generalizable elsewhere. However, treating clinicians within this system are unlikely to differ substantially from clinicians in other systems in diagnostic acumen, treatment strategies, or documentation habits. This validation study was limited to outcomes associated with an IP or ED visit or death and the results cannot be extrapolated to patients without these types of medical visits. Further, these outcomes were measured among adults with diabetes and may not be applicable to other populations.

\section{Conclusion}

Automated healthcare data can be useful when identifying hyperkalemia-associated adverse outcomes. Potassium laboratory test results provide incremental value in identifying such outcomes. Because both false positives and negatives occur with coded hyperkalemia diagnosis, medical record validation is necessary.

\section{Acknowledgments}

The work contained in this manuscript was conducted under: "Diabetes and Drug-Associated Hyperkalemia: Effect of Laboratory Monitoring" supported by the National Institute of Diabetes and Digestive and Kidney Diseases (NIDDK) Award Number R21DK075076. The content is solely the responsibility of the authors and does not necessarily represent the official views of the National Institute of Diabetes And Digestive and Kidney Diseases or the National Institutes of Health.

We thank Edward Quinn for developing the electronic abstraction databases and Colleen Ross for completing the analytic work necessary to identify the study subjects and outcomes from the administrative data.

\section{References}

1. Palmer BF. Angiotensin-converting enzyme inhibitors and angiotensin receptor blockers: what to do if the serum creatinine and/or serum potassium concentration rises. Nephrol Dial Transplant. 2003; 18:1973-1975. [PubMed: 13679467]

2. Palmer BF. Managing hyperkalemia caused by inhibitors of the renin-angiotensin-aldosterone system. N Engl J Med. 2004; 351:585-592. [PubMed: 15295051]

3. Juurlink DN, Mamdani MM, Lee DS, et al. Rates of hyperkalemia after publication of the randomized aldactone evaluation study. N Engl J Med. 2004; 351:543-551. [PubMed: 15295047]

4. Ahuja TS, Freeman D Jr, Mahnken JD, Agraharkar M, Siddiqui M, Memon A. Predictors of the development of hyperkalemia in patients using angiotensin-converting enzyme inhibitors. Am J Nephrol. 2000; 20(4):268-272. [PubMed: 10970978]

5. Desai AS, Swedberg K, McMurray JJV, et al. Incidence and predictors of hyperkalemia in patients with heart failure: An analysis of the CHARM program. J Am Coll Cardiol. 2007; 50(20):1959_ 1966. [PubMed: 17996561]

6. Ramadan FH, Masoodi N, El-Solh AA. Clinical factors associate with hyperkalemia in patients with congestive heart failure. J Clin Pharm Therapeutics. 2005; 30:233-239.

7. Juurlink DN, Mamdani M, Kopp A, Laupacis A, Redelmeier DA. Drug-drug interactions among elderly patients hospitalized for drug toxicity. JAMA. 2003; 289:1652-1658. [PubMed: 12672733]

8. Schaefer TJ, Wolford RW. Disorders of potassium. Emerg Med Clin N Am. 2005; 23:723-747.

9. Williams ME. Endocrine crises. Hyperkalemia. Critical Care Clinics. 1991; 7(1):155-174.

[PubMed: 2007212] 
10. Raebel MA, Ross C, Cheetham C, et al. Increasingly restrictive definitions of hyperkalemia outcomes in a database study: Effect on incidence estimates. Pharmacoepidemiol Drug Saf. 2010; 19(1):19-25. [PubMed: 19937982]

11. Raebel MA, Ross C, Xu S, et al. Diabetes and Drug-Associated Hyperkalemia: Effect of Serum Potassium Monitoring. J Gen Intern Med. 2010; 25(4):326-333.10.1007/s11606-009-1228-x [PubMed: 20087674]

12. Xu S, Ross C, Raebel MA, Shetterly S, Blanchette C, Smith D. Use of stabilized inverse propensity scores as weights to directly estimate relative risk and its confidence intervals. Value in Health. 2010; 13(2):273-277. [PubMed: 19912596]

13. Raebel MA, McClure DL, Simon SR, et al. Laboratory monitoring of angiotensin converting enzyme inhibitors and angiotensin receptor blockers in ambulatory patients. Pharmacoepidemiol Drug Saf. 2007; 16:55-64. [PubMed: 16470693]

14. Hanley JA, Lippman-Hand A. If nothing goes wrong, is everything all right? JAMA. 1983; 249(13):1743-1745. [PubMed: 6827763]

15. Daly L. Simple SAS macros for the calculation of exact Binomial and Poisson confidence limits. Comput Biol Med. 1992; 22(5):351-361. [PubMed: 1424580] 


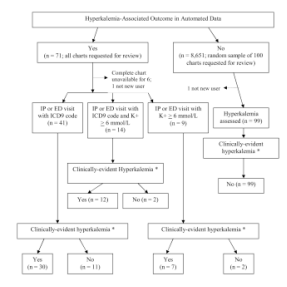

Figure. Hyperkalemia-Associated Outcomes in Automated Data and Medical Record Documentation of Hyperkalemia-Associated Clinical Signs, Treatment, and/or Change in Clinical Plan

* See text for definitions 


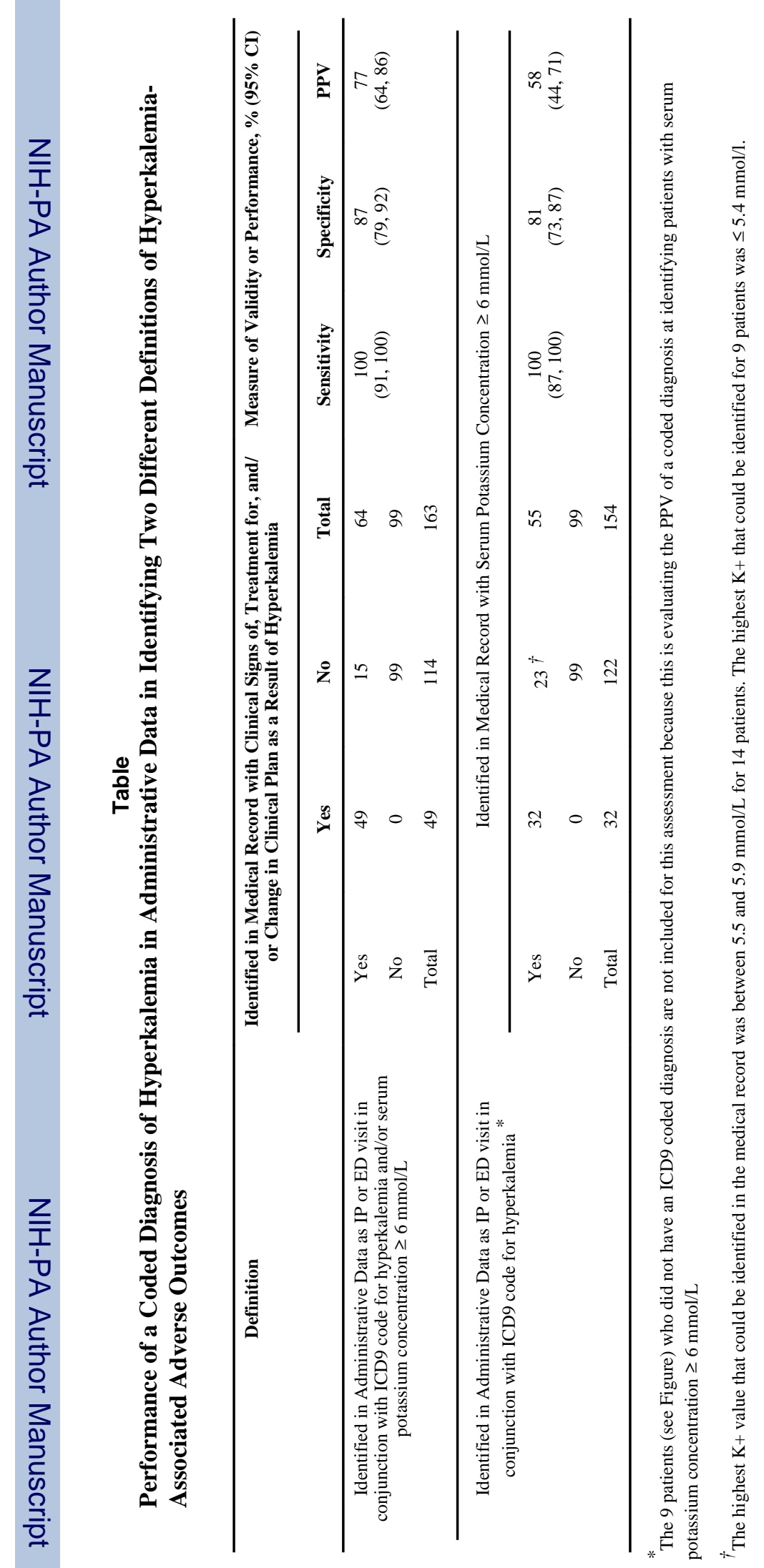

Pharmacoepidemiol Drug Saf. Author manuscript; available in PMC 2011 November 1. 